\title{
5 LITERACIA EM SAÚDE MENTAL SOBRE ABUSO DE ÁLCOOL: UM ESTUDO COM ADOLESCENTES PORTUGUESES
}

\author{
| Amorim Rosa ${ }^{1}$; Luís Loureiro ${ }^{2}$; Carlos Sequeira ${ }^{3}$ |
}

\section{RESUMO}

CONTEXTO: A adolescência configura um período conturbado em torno da busca da identidade, indiciando um processo de vulnerabilidade em relação ao início das perturbações mentais, mas também ao abuso de álcool. A esta vulnerabilidade associa-se uma forte relutância e atrasos na procura de ajuda profissional que, para além de agravar o problema, poderão comprometer o potencial de desenvolvimento dos adolescentes. Por outro lado, o uso precoce de álcool implica um maior risco de dependência na idade adulta.

OBJETIVO: Caraterizar a literacia em saúde mental sobre abuso de álcool em adolescentes.

METODOLOGIA: Estudo exploratório descritivo. Foi aplicada a MentaHLiS-AA a uma amostra de 255 adolescentes (159 raparigas e 96 rapazes), com idades entre os 10 e os 18 anos $(\mathrm{M}=14.38, \mathrm{DP}=2.20)$, estudantes do $2^{\circ}$ e $3^{\circ}$ ciclos e secundário, em escolas públicas do distrito de Coimbra. Calcularam-se as estatísticas resumidas apropriadas e as frequências absolutas e percentuais.RESULTADOS: Verificou-se que 25,5\% dos participantes não reconheceram o problema apresentado na vinheta. Nos outros componentes, os níveis de literacia são modestos, verificando-se a preferência por fontes informais de ajuda e a opção por estratégias de apoio social e encaminhamento passivo para ajudar os pares com problemas de saúde mental.

CONCLUSÃO: Verificaram-se défices em alguns aspetos da literacia sobre o abuso de álcool em adolescentes, sendo necessária uma intervenção nesta área, sobretudo através do desenvolvimento e implementação de programas de promoção da literacia nas escolas.

PALAVRAS-CHAVE: Literacia; Saúde mental; Adolescentes; Abuso de álcool

\section{RESUMEN}

“Alfabetización en salud mental sobre abuso de alcohol: Un estudio con adolescentes portugueses"

CONTEXTO: La adolescencia configura un período conturbado en torno a la búsqueda de la identidad, indiciando un proceso de vulnerabilidad en relación al inicio de las perturbaciones mentales, pero también al abuso de alcohol. A esta vulnerabilidad se asocia una fuerte reticencia y retrasos en la demanda de ayuda profesional que, además de agravar el problema, podrán comprometer el potencial de desarrollo de los adolescentes. Por otro lado, el uso precoz de alcohol implica un mayor riesgo de dependencia en la edad adulta.

OBJETIVO: Caracterizar la alfabetización en salud mental sobre abuso de alcohol en adolescentes.

METODOLOGÍA: Estudio exploratorio descriptivo. Se aplicó la MentaHLiS-AA a una muestra de 255 adolescentes (159 niñas y 96 niños), con edades entre los 10 y los 18 años $(\mathrm{M}=14.38, \mathrm{DE}=2.20)$, estudiantes de $2^{\circ}$ y $3^{\circ}$ ciclos y secundario en escuelas públicas del distrito de Coimbra. Se calcularon las estadísticas resumidas apropiadas y las frecuencias absolutas y porcentuales.

RESULTADOS: Se verificó que el 25,5\% de los participantes no reconoció el problema presentado en la viñeta. En los otros componentes, los niveles de alfabetización son modestos, verificándose la preferencia por fuentes informales de ayuda y la opción por estrategias de apoyo social y encaminamiento pasivo para ayudar a los pares con problemas de salud mental. CONCLUSIONES: Se verificaron déficit en algunos aspectos de la alfabetización sobre el abuso de alcohol en los adolescentes portugueses, siendo necesaria una intervención en esta área, sobre todo a través del desarrollo e implementación de programas de promoción de la alfabetización en las escuelas.

\section{DESCRIPTORES: Alfabetización; Salud mental; Adolescentes; Abuso de alcohol}

\section{ABSTRACT \\ "Mental health literacy on alcohol abuse: A study with Portu- guese adolescents"}

BACKGROUND: Adolescence is a troubled period around the search for identity, indicating a process of vulnerability to the onset of mental disorders, but also to alcohol abuse. This vulnerability is associated with a strong reluctance and delays in professional help seeking, which, in addition to worsening the problem, could compromise the adolescents' potential for development. On the other hand, the early use of alcohol implies a greater risk of dependence in adulthood.

AIM: Characterize mental health literacy on alcohol abuse in adolescents. METHODS: Exploratory descriptive study. The MentaHLiS-AA was applied to a sample of 255 adolescents (159 girls and 96 boys), aged between 10 and 18 years $(M=14.38, S D=2.20)$, attending schools in the 2 nd and 3 rd cycle and secondary education in Coimbra. The appropriate summary statistics and the absolute and percentage frequencies were calculated.

RESULTS: We found that $25.5 \%$ of participants did not recognize the problem presented in the vignette. In the other components, literacy levels are modest, with preference for informal sources of help and choice for social support and passive referral strategies to help peers with mental health problems.

CONCLUSIONS: There are deficits in literacy on alcohol abuse among adolescents, requiring intervention in this area, especially through the development and implementation of programs to promote mental health literacy in schools.

\section{KEYWORDS: Literacy; Mental health; Adolescents; Alcohol abuse}

Submetido em 18-12-2016

Aceite em 20-04-2017

1 Doutor; Professor Adjunto na Escola Superior de Enfermagem de Coimbra, Rua 5 de Outubro, Apartado 7001, 3046-851 Coimbra, Portugal, amorim@esenfc.pt 2 Doutor; Professor Adjunto na Escola Superior de Enfermagem de Coimbra, 3046-851 Coimbra, Portugal, lloureiro@esenfc.pt

3 Doutor; Professor Coordenador na Escola Superior de Enfermagem do Porto, 4200-072 Porto, Portugal, carlossequeira@esenf.pt 


\section{INTRODUÇÃO}

A adolescência pode configurar um período conturbado em torno da busca da identidade. As transições, definições e experiências que ocorrem nesta fase, em alguns casos, tornam-se particularmente desestabilizadoras, causando sofrimento psíquico e determinando uma vulnerabilidade acrescida para a exposição ao abuso de substâncias, como é o caso do álcool (Rosa, Loureiro e Sequeira, 2016). Neste período, é comum ocorrerem oscilações no estado emocional, envolvendo sentimentos de tristeza, revolta e depressão, podendo o adolescente procurar no consumo de álcool, uma forma de 'mascarar' as situações conflituantes, internas ou sociais (Tavares, Reinaldo, Villa, Henriques e Pereira, 2017). Por outro lado, a necessidade de se sentir aceite pelos pares e de definir a sua identidade, podem também ser fatores determinantes da experimentação (Ribeiro, Pereira, Wiesl, Silva e Saldanha, 2017).

Estudos epidemiológicos demonstram que o consumo de álcool é uma das principais preocupações de saúde pública, particularmente em relação aos adolescentes, porque é nesta fase que geralmente ocorrem as primeiras experiências de consumo (Atanázio, Santos, Dionísio, Silva e Saldanha, 2013), e porque constitui o maior fator de risco para a saúde dos adolescentes (Ferreira e Torgal, 2010 citados por Faria e Martins, 2016).

Os estudos indicam que quanto mais precoce é a fase de experimentação, maior o risco de abuso e dependência na idade adulta (Wandekoken, Vicente e Siqueira, 2011).

Neste sentido, atendendo a que o uso precoce de álcool é altamente prevalente entre os adolescentes e que o seu consumo tem sido associado a alterações na estrutura cerebral, função e neuro cognição, além de inúmeras repercussões negativas no campo social (Tavares et al., 2017), têm sido desenvolvidas intervenções no âmbito da educação para a saúde, visando a prevenção do consumo precoce e do seu uso inadequado, alertando para os riscos, consequências e efeitos individuais e coletivos.

Estas intervenções, se ancoradas no conceito de literacia em saúde mental (LSM), tendem a ser eficazes, pois implicam um conhecimento voltado para a ação (Jorm, 2012; Loureiro et al., 2013a), traduzido em comportamentos de procura de ajuda, que estão relacionados de forma direta com os níveis de LSM (Chong et al., 2016; Jorm, 2012).
A LSM, definida como os conhecimentos e crenças acerca das perturbações mentais que facilitam o seu reconhecimento, prevenção e/ou gestão, engloba um conjunto de componentes interligadas: a) conhecimento de como prevenir as perturbações mentais; $b$ ) reconhecimento do despoletar da doença; c) conhecimento das opções de ajuda e tratamentos disponíveis; d) conhecimento de estratégias de autoajuda para os problemas menos graves; e e) competências para prestar a primeira ajuda, a outras pessoas em situação de crise ou em desenvolvimento de uma perturbação mental (Jorm, 2012).

Assim, apesar de alguns estudos mostrarem que o abuso de álcool é uma das perturbações mais frequentemente reconhecidas (Chong et al., 2016; Picco et al., 2017), de um modo geral os níveis de LSM são modestos pelo que é imperativo que os profissionais de saúde, sobretudo os enfermeiros, concebam e implementem programas de educação e sensibilização para a saúde mental, empoderando os adolescentes com as habilidades e conhecimentos necessários para identificar os sinais e sintomas do problema, promovendo comportamentos de procura de ajuda precoces, o que poderá reduzir a carga de doença associada (Bor, 2015).

Este estudo tem como objetivo caraterizar a LSM sobre abuso de álcool em adolescentes.

\section{METODOLOGIA}

\section{Participantes}

Estudo de natureza quantitativa, nível exploratório descritivo, realizado com uma amostra de 255 adolescentes, com idades compreendidas entre os 10 e os 18 anos, a frequentar o $2^{\circ}$ e $3^{\circ}$ ciclos e secundário, em 11 escolas do distrito de Coimbra, selecionadas aleatoriamente através do software Random Integer Generator. Em cada escola foi selecionada uma turma correspondente a cada um dos ciclos de estudos. A amostra foi constituída pelos adolescentes que acederam participar no estudo após informação dos seus objetivos e autorização dos pais ou encarregados de educação.

\section{Instrumento de Colheita de Dados}

O instrumento utilizado (MentaHLiS-AA) é constituído por cinco subescalas que avaliam os componentes da LSM, utilizando diferentes formatos de resposta. A $1^{\text {a }}$ parte inclui instruções de preenchimento e questões de caraterização sociodemográfica (género, idade e escolaridade dos participantes e habilitações literárias dos pais). 
A $2^{\text {a }}$ parte compreende as subescalas relativas aos componentes da LSM, sendo as questões ancoradas numa vinheta clínica, retratando o caso de um/a adolescente (Pedro/Francisca) com critérios para abuso de álcool, segundo o DSM-IV-TR, adaptada a partir da versão original do Survey of Mental Health Literacy in Young People - Interview Version (Jorm, 2000). Das cinco subescalas que compõem a MentaHLiSAA, três avaliam componentes específicos relacionados com o problema apresentado na vinheta: I - 'Reconhecimento do problema'; II - 'Recursos e opções de ajuda'; e III - 'Crenças e intenções para prestar a primeira ajuda', enquanto as restantes duas avaliam dimensões e atributos genéricos: IV - 'Barreiras e facilitadores na procura de ajuda'; e V - 'Estilos de vida e comportamentos de saúde'. Neste estudo, tendo em conta o seu objetivo, foram utilizados unicamente os resultados das subescalas I, II e III que avaliam os componentes específicos relacionadas com o "Abuso de álcool".

A subescala 'I' avalia a capacidade dos adolescentes para reconhecer o problema, selecionando as respostas numa escala de escolha múltipla, composta por 10 rótulos com diferentes níveis de adequação à situação apresentada. Os valores do alfa de Cronbach mostram um bom ajustamento do modelo: dimensão 1 (.670); dimensão 2 (.369); média com base no autovalor médio (.558). A subescala 'II' $(\alpha=.789)$ é composta por nove opções de resposta à questão "Como consideras que cada uma das seguintes pessoas poderia ajudar o/a Pedro/Francisca?" É solicitado aos participantes que pontuem as diferentes opções apresentadas numa escala de 1 (prejudicaria muito) a 5 (ajudaria muito). Por fim, a subescala 'III' $(\alpha=.756)$ inclui 7 opções de resposta à questão "O que farias para ajudar o/a Pedro/Francisca?” É pedido aos participantes que assinalem numa escala de 1 (discordo muito) a 5 (concordo muito), em que medida concordam com cada ação apresentada.

O conteúdo da totalidade dos itens e a vinheta utilizada apresentam-se em detalhe no estudo de construção e validação do instrumento (Rosa et al., 2016).

\section{Procedimento}

Os dados foram recolhidos nos meses de abril e maio de 2014. Os questionários foram aplicados pelos professores diretores de turma, em sala de aula, em sessões coletivas, num período especificamente disponibilizado para o efeito. O tempo de resposta do questionário situou-se entre os 15 e os 20 minutos.

\section{Aprovação Ética}

$\mathrm{O}$ estudo e o instrumento foram aprovados pela Direcção-Geral de Educação - Gabinete de Projetos Educativos do Ministério da Educação do Governo Português (n. ${ }^{\circ}$ 0404700001) e pela Comissão de Ética da Unidade de Investigação em Ciências da Saúde - Enfermagem (UICISA-E) da Escola Superior de Enfermagem de Coimbra (P187-10-2013). Dadas as caraterísticas da amostra, todos os participantes e encarregados de educação assinaram um termo de consentimento informado, após serem informados sobre os objetivos do estudo.

\section{Análise Estatística}

Os dados foram analisados com o software IBM SPSS22. Como se trata de um estudo exploratório descritivo, foram calculadas as estatísticas resumo adequadas e as frequências absolutas e percentuais de modo a responder ao objetivo do estudo, utilizando-se tabelas de frequência simples e compostas.

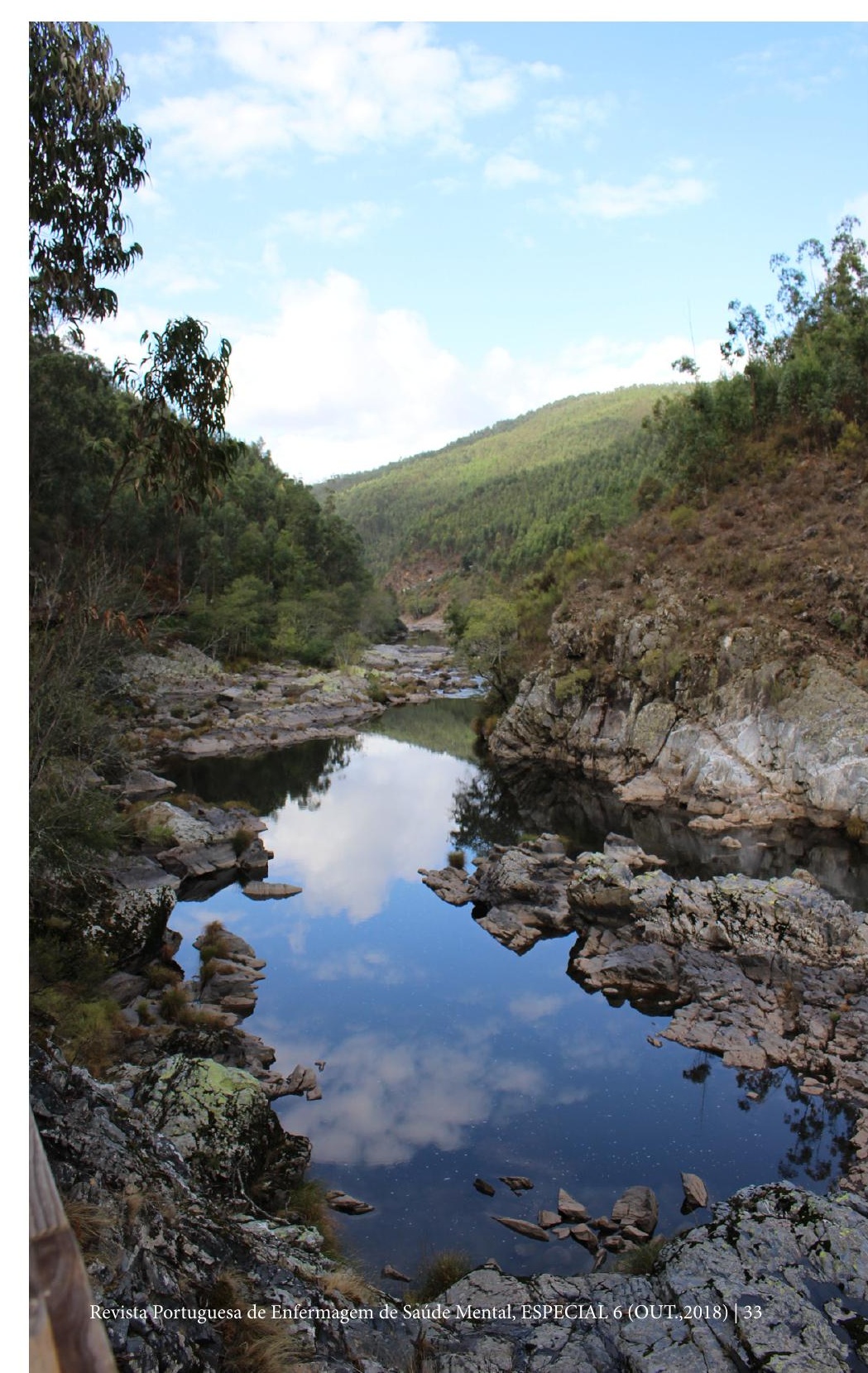




\section{RESULTADOS}

Participaram no estudo 255 adolescentes, sendo 37,6\% do género masculino e $62,4 \%$ do género feminino. As idades variam entre os 10 e os 18 anos, sendo a média de 14,38 anos e o desvio padrão de 2,2. A classe modal é a dos 13-15 anos com 122 indivíduos, correspondendo a uma percentagem de $47,8 \%$. A maioria dos adolescentes frequenta o $3^{\circ}$ ciclo (55,3\%), 32,2\% frequentam o ensino secundário e $12,5 \%$, o $2^{\circ}$ ciclo.

Os pais têm níveis de escolaridade entre o $1^{\circ}$ ciclo e o mestrado, verificando-se uma distribuição semelhante, na sua maioria nos quatro primeiros níveis de ensino ( $1^{\circ}$ ciclo; $2^{\circ}$ ciclo; $3^{\circ}$ ciclo, e ensino secundário), correspondendo a $86,3 \%$ dos pais e $72,9 \%$ das mães (Tabela 1).

Tabela 1 - Distribuição das frequências absolutas e percentagens dos participantes em função das variáveis de caraterização sócio demográfica $(\mathrm{N}=255)$

\begin{tabular}{|c|c|c|}
\hline Variáveis & $\mathbf{N}$ & $\%$ \\
\hline \multicolumn{3}{|l|}{ Sexo: } \\
\hline Masculino & 96 & 37,6 \\
\hline Feminino & 159 & 62,4 \\
\hline \multicolumn{3}{|l|}{ Idade: } \\
\hline $10-12$ & 52 & 20,4 \\
\hline $13-15$ & 122 & 47,8 \\
\hline $16-18$ & 81 & 31,8 \\
\hline \multicolumn{3}{|l|}{ Escolaridade: } \\
\hline $2^{\circ}$ Ciclo & 32 & 12,5 \\
\hline $3^{\circ}$ Ciclo & 141 & 55,3 \\
\hline Secundário & 82 & 32,2 \\
\hline \multicolumn{3}{|l|}{ Escolaridade pai: } \\
\hline $1^{\circ}$ Ciclo & 40 & 15,7 \\
\hline $2^{\circ}$ Ciclo & 51 & 20,0 \\
\hline $3^{\circ}$ Ciclo & 67 & 26,3 \\
\hline Secundário & 62 & 24,3 \\
\hline Bacharelato/Licenciatura & 27 & 10,6 \\
\hline Outro & 8 & 3,1 \\
\hline \multicolumn{3}{|l|}{ Escolaridade mãe: } \\
\hline $1^{\circ}$ Ciclo & 20 & 7,8 \\
\hline $2^{\circ}$ Ciclo & 34 & 13,3 \\
\hline $3^{\circ}$ Ciclo & 62 & 24,3 \\
\hline Secundário & 70 & 27,5 \\
\hline Bacharelato/Licenciatura & 60 & 23,5 \\
\hline Outro & 9 & 3,6 \\
\hline
\end{tabular}

Sendo a MentaHLiS-AA um instrumento que avalia diversos componentes da LSM, os resultados serão apresentados de forma independente para cada componente.

\section{Reconhecimento do Problema (Abuso de Álcool)}

No componente 'Reconhecimento do problema', a opção mais assinalada foi "Tem problemas com a bebida" com 74,5\%, seguida de "Vício" (61,6\%), "Dificuldades em se controlar" (49,8\%), e "Falta de responsabilidade" com 41,6\%. Todas as outras opções de resposta tiveram percentagens relativamente baixas. Contudo, é de realçar também que $25,5 \%$ dos participantes não foram capazes de identificar o problema, mesmo perante os critérios claros apresentados na vinheta (Tabela 2).

Tabela 2 - Distribuição das frequências absolutas e percentuais para as categorias do componente

'Reconhecimento do problema' $(\mathrm{N}=255)$

\begin{tabular}{|l|c|c|c|c|}
\hline \multirow{2}{*}{$\begin{array}{l}\text { Reconhecimento do } \\
\text { problema }\end{array}$} & \multicolumn{2}{|c|}{ Sim } & \multicolumn{2}{c|}{ Não } \\
\cline { 2 - 5 } & $\mathrm{N}$ & $\%$ & $\mathrm{~N}$ & $\%$ \\
\hline Anda nervoso e triste & 59 & 23,1 & 196 & 76,9 \\
\hline Tem problemas afetivos & 52 & 20,4 & 203 & 79,6 \\
\hline Tem falta de confiança & 72 & 28,2 & 183 & 71,8 \\
\hline Está revoltado & 88 & 34,5 & 167 & 65,5 \\
\hline Está a reagir a uma perda & 31 & 12,2 & 224 & 87,8 \\
\hline Tem baixa autoestima & 67 & 26,3 & 188 & 73,7 \\
\hline $\begin{array}{l}\text { Tem dificuldades em se } \\
\text { controlar }\end{array}$ & 127 & 49,8 & 128 & 50,2 \\
\hline Tem um vício & 157 & 61,6 & 98 & 38,4 \\
\hline $\begin{array}{l}\text { Tem falta de responsabi- } \\
\text { lidade }\end{array}$ & 106 & 41,6 & 149 & 58,4 \\
\hline $\begin{array}{l}\text { Tem problemas com a } \\
\text { bebida }\end{array}$ & 190 & 74,5 & 65 & 25,5 \\
\hline
\end{tabular}

\section{Recursos e Opções de Ajuda}

Quanto aos diferentes recursos e opções de ajuda para a situação apresentada na vinheta (Tabela 3), verificase que os adolescentes consideram como ajudas mais úteis, os amigos de confiança $(95,3 \%)$ e os familiares (92,5\%), mas também alguns profissionais de saúde, destacando-se o psicólogo (85,5\%), o psiquiatra $(72,5 \%)$ e o enfermeiro especialista de saúde mental e psiquiatria $(60,8 \%)$. Salienta-se também a ambivalência relativa à capacidade de ajuda de alguns profissionais, sendo esta mais significativa relativamente ao médico de família e aos professores, sendo até considerados prejudiciais por $6,7 \%$ e $11,4 \%$ dos adolescentes, respetivamente (Tabela 3 ). 
Tabela 3 - Distribuição das frequências absolutas e percentuais para as categorias do componente

'Recursos e opções de ajuda' ( $\mathrm{N}=255)$

\begin{tabular}{|l|l|l|l|l|l|l|}
\hline \multirow{2}{*}{$\begin{array}{l}\text { Recursos e } \\
\text { opções de } \\
\text { ajuda }\end{array}$} & \multicolumn{2}{|l|}{$\begin{array}{l}\text { Prejudica } \\
\text { Prejudica muito }\end{array}$} & \multicolumn{2}{|l|}{$\begin{array}{l}\text { Não ajuda nem } \\
\text { prejudica }\end{array}$} & \multicolumn{2}{|l|}{$\begin{array}{l}\text { Ajuda } \\
\text { Ajuda muito }\end{array}$} \\
\cline { 2 - 7 } & $\mathrm{N}$ & $\%$ & $\mathrm{~N}$ & $\%$ & $\mathrm{~N}$ & $\%$ \\
\hline $\begin{array}{l}\text { Amigos de } \\
\text { confiança }\end{array}$ & 1 & 0,4 & 0,4 & 4,3 & 243 & 95,3 \\
\hline Familiares & 2 & 0,8 & 0,8 & 6,7 & 236 & 92,5 \\
\hline $\begin{array}{l}\text { Grupos de } \\
\text { autoajuda }\end{array}$ & 12 & 4,7 & 4,7 & 10,6 & 216 & 84,7 \\
\hline Psicólogo & 6 & 2,4 & 2,4 & 12,2 & 218 & 85,5 \\
\hline Psiquiatra & 10 & 3,9 & 3,9 & 23,5 & 185 & 72,5 \\
\hline $\begin{array}{l}\text { Enfermeiro } \\
\text { especialista }\end{array}$ & 12 & 4,7 & 4,7 & 34,5 & 155 & 60,8 \\
\hline $\begin{array}{l}\text { Médico de } \\
\text { família }\end{array}$ & 17 & 6,7 & 6,7 & 33,7 & 152 & 59,6 \\
\hline Professores & 29 & 11,4 & 11,4 & 34,9 & 137 & 53,7 \\
\hline
\end{tabular}

Ao nível das crenças e intenções para prestar a primeira ajuda (Tabela 4), verifica-se que os participantes concordam mais com estratégias de apoio social e encaminhamento passivo, considerando mais útil ouvir e compreender (95,7\%); apoiar e animar (94,1\%); e acompanhar/não abandonar (92,5\%), desvalorizando o encaminhamento ativo com envolvimento de adultos, verificando-se que apesar de $87,1 \%$ dos participantes concordarem com o encaminhamento para um profissional de saúde especializado, só cerca de metade concordam com o encaminhamento para o médico de família $(53,3 \%)$ ou para um professor de confiança $(52,5 \%)$, verificando-se ainda que $12,2 \%$ dos participantes discordam do encaminhamento para estes profissionais.

Tabela 4 - Distribuição das frequências absolutas e percentuais para as categorias do componente 'Crenças e intenções para prestar a primeira ajuda' $(\mathrm{N}=255)$

\begin{tabular}{|l|l|l|l|l|l|l|}
\hline \multirow{2}{*}{$\begin{array}{l}\text { Crenças e intenções para } \\
\text { prestar a primeira ajuda }\end{array}$} & \multicolumn{2}{|l|}{$\begin{array}{l}\text { Discordo } \\
\text { Discordo } \\
\text { muito }\end{array}$} & \multicolumn{2}{l|}{$\begin{array}{l}\text { Não con- } \\
\text { cordo } \\
\text { nem discordo }\end{array}$} & \multicolumn{2}{l|}{$\begin{array}{l}\text { Concordo } \\
\text { Concordo } \\
\text { muito }\end{array}$} \\
\cline { 2 - 8 } & $\mathrm{N}$ & $\%$ & $\mathrm{~N}$ & $\%$ & $\mathrm{~N}$ & $\%$ \\
\hline Dar-lhe apoio e animá-lo/a & 5 & 2,0 & 10 & 3,9 & 240 & 94,1 \\
\hline $\begin{array}{l}\text { Ouvi-lo/a e tentar } \\
\text { compreendê-lo/a }\end{array}$ & 2 & 0,8 & 9 & 3,5 & 244 & 95,7 \\
\hline $\begin{array}{l}\text { Encaminhá-lo/a para um } \\
\text { prof. saúde especializado }\end{array}$ & 6 & 2,4 & 27 & 10,6 & 222 & 87,1 \\
\hline $\begin{array}{l}\text { Acompanhá-lo/a à con- } \\
\text { sulta, não o/a abandonar }\end{array}$ & 5 & 2,0 & 14 & 5,5 & 236 & 92,5 \\
\hline $\begin{array}{l}\text { Falar com os familiares } \\
\text { (...) estejam mais atentos }\end{array}$ & 11 & 4,3 & 26 & 10,2 & 218 & 85,5 \\
\hline $\begin{array}{l}\text { Encaminhá-lo/a para um } \\
\text { professor de confiança }\end{array}$ & 31 & 12,2 & 90 & 35,2 & 134 & 52,5 \\
\hline $\begin{array}{l}\text { Encaminhá-lo/a para o } \\
\text { médico de família }\end{array}$ & 31 & 12,2 & 88 & 34,5 & 136 & 53,3 \\
\hline
\end{tabular}

\section{DISCUSSÃO}

No presente estudo os autores pretenderam caraterizar a LSM sobre abuso de álcool em adolescentes, incidindo sobre os componentes: reconhecimento da perturbação; recursos e opções de ajuda; e crenças e intenções para prestar a primeira ajuda.

De um modo geral a literatura aponta a adolescência como um período crítico durante o qual ocorrem as primeiras experiências de consumo de substâncias, existindo múltiplos fatores relacionados com esta fase desenvolvimental que indiciam um processo de vulnerabilidade do adolescente em relação ao uso e abuso de álcool. É, pois, fundamental que se realize um trabalho específico de promoção da LSM dos adolescentes, fator determinante para a minimização dos riscos associados à falta de informação, que é um dos maiores obstáculos para a intervenção precoce (Tomé, Matos, Gomes, Camacho e Gaspar, 2017).

Dos vários componentes da LSM, o reconhecimento do problema emerge como um fator inicial importante, pois é o primeiro passo para a procura de ajuda profissional (Gulliver, Griffiths \& Christensen, 2010). Um reconhecimento precoce reduz de forma significativa o intervalo de tempo que medeia entre o surgimento dos sintomas e o acesso aos cuidados de saúde, melhorando a qualidade de vida e os resultados em saúde, tanto do indivíduo como da comunidade (Jorm, 2012; Loureiro et al., 2013b). Depreende-se, então, que défices de LSM, traduzidos na incapacidade de reconhecer os diferentes transtornos e as fontes de ajuda disponíveis, bem como crenças erradas sobre como ajudar os outros, conduzirão ao consequente adiamento da procura de ajuda.

Os resultados deste estudo mostram a complexidade do fenómeno do uso e abuso de álcool e do seu reconhecimento como 'perturbação', configurando predominantemente um desconhecimento ou visão difusa do problema (Rosa et al., 2016). Por um lado, a maioria dos adolescentes reconhece a situação apresentada na vinheta como um problema de abuso de álcool, contudo, uma percentagem semelhante classifica a situação como um "Vício".

Esta diferença não deve ser entendida como uma mera questão semântica, isto porque o termo 'vício' poderá traduzir uma perceção ou visão estereotipada, que no caso do abuso de álcool está muito enraizada na sociedade, podendo dificultar ou impedir que o problema seja percecionado como doença mental (Loureiro, 2013a). 
Por outro lado, a associação do abuso de álcool com outros fatores como falta de responsabilidade e dificuldades de controlo, caraterísticas de um período de transição crítica ou crise própria da adolescência, é também uma ideia partilhada na sociedade, podendo conduzir a uma desvalorização do problema e constituir uma barreira aos comportamentos de procura e oferta de ajuda (Reavley \& Jorm, 2011), bem como à implementação de programas de prevenção (Rickwood, Deane, Wilson \& Ciarrochi, 2005).

Relativamente ao componente 'Recursos e opções de ajuda', verifica-se uma clara preferência dos adolescentes por fontes de ajuda informais. Este facto releva pela perceção de que os adolescentes tendem a desvalorizar as ajudas profissionais, preferindo apoiar-se nos pares e noutras fontes informais como a família, o que poderá retardar de forma significativa o acesso a ajuda especializada.

No entanto, a valorização das fontes informais é importante, tanto pelo apoio prestado, como pelo facto de que, muitas vezes, é pela sugestão de amigos e familiares que os adolescentes procuram ajuda (Loureiro et al., 2013b). Porém, salienta-se a sua importância no apoio, mas também a sua insuficiência na ajuda, podendo mesmo ser prejudiciais se a informação prestada for imprecisa e sem utilidade, tendo como consequência o agravamento dos sintomas e o atraso na procura de ajuda especializada (Power, Eiraldi, Clarke, Mazzuca \& Krain, 2005; Jorm, 2012, citados por Loureiro, 2013b).

Numa segunda linha de importância, surge o psicólogo, o psiquiatra e o médico de família, que enquanto recurso de ajuda próximo e acessível, são a "porta de entrada" nos serviços de saúde, cabendo-lhe a responsabilidade de referenciação das situações para níveis mais diferenciados de cuidados. No entanto, alguns estudos (e.g. Jorm, Wright \& Morgan, 2007) têm mostrado que as pessoas com problemas de saúde mental, sobretudo os adolescentes, desvalorizam o papel do médico relativamente a outros profissionais de saúde, ainda que, particularmente o médico de família, seja considerado uma das principais fontes de ajuda profissional útil, conjuntamente com o psicólogo (Loureiro et al., 2013b).

Contudo, os profissionais dos cuidados de saúde primários, onde se incluem o médico e o enfermeiro de família, não têm formação adequada pelo que os problemas de saúde mental geralmente não são diagnosticados ou não são detetados (Bor, 2015).
Só cerca de metade dos adolescentes reconhecem o professor como uma ajuda útil ou muito útil, e mais de $10 \%$ consideram-no prejudicial. Este dado é preocupante, no entanto, poderá ser justificado pelo facto de os adolescentes considerarem que a partilha de problemas pessoais com os professores pode comprometer o seu desempenho e aproveitamento escolar, podendo também estar em causa questões de confidencialidade (Rickwood et al., 2005).

Finalmente, no componente 'Crenças e intenções para prestar a primeira ajuda', os adolescentes consideram as estratégias de apoio social e encaminhamento passivo como as mais importantes (e.g. ouvir, apoiar, compreender e acompanhar), mas também o encaminhamento ativo com envolvimento de adultos (e.g. encaminhar para ajuda profissional), o que está claramente de acordo com as principais linhas orientadoras dos procedimentos de primeira ajuda em saúde mental (Loureiro, et al., 2013b). De realçar que, também nesta dimensão, o encaminhamento para um professor de confiança é, em simultâneo, a opção com a qual menos adolescentes concordam $(52,5 \%)$ e aquela com que mais discordam (12,2\%).

\section{CONCLUSÕES E IMPLICAÇÕES PARA A PRÁTI- $\mathrm{CA}$}

Os resultados deste estudo, o primeiro realizado com recurso a este instrumento, revelam que o nível de LSM dos adolescentes é modesto na generalidade dos componentes analisados, pelo que a literacia pode e deve ser melhorada, sobretudo tendo em conta que o abuso/dependência de álcool é uma das perturbações mentais mais graves e de maior prevalência, e que as primeiras experiências de consumo de álcool ocorrem geralmente no início da adolescência, observando-se simultaneamente um consumo excessivo ou abusivo antes dos 18 anos. A estes fatores acresce ainda o facto de a adolescência configurar um período durante o qual as experiências vivenciadas se podem tornar particularmente desestabilizadoras, causando sofrimento psíquico e determinando uma vulnerabilidade acrescida para a exposição ao abuso de álcool.

Assim, tendo em vista uma participação mais ativa dos adolescentes nos comportamentos de saúde, compete aos enfermeiros desenvolver e implementar ações educativas integradas num contexto mais vasto de promoção da saúde, incidindo sobre os diversos componentes da LSM e desenvolvidas nos contextos educativos e escolares. 
Para tal, devem socorrer-se de ações específicas de informação e treino de competências, capazes de promover a LSM e empoderar os adolescentes para a tomada de decisão e adoção de comportamentos de saúde, sobretudo de procura de ajuda profissional para os problemas de saúde mental.

Pelo exposto, torna-se clara a importância deste estudo e a sua relevância para a prática de Enfermagem, visando a promoção de uma saúde mental positiva nos adolescentes. Baixos níveis de LSM implicam dificuldades significativas em aspetos como por exemplo, a compreensão das doenças mentais e dos seus sintomas, a procura de ajuda adequada, a capacidade de ajudar os pares com problemas de saúde mental, e a identificação dos recursos disponíveis ao nível da comunidade, tendo como consequência, entre outros aspetos, um maior número de hospitalizações, aumento dos custos com cuidados de saúde mental e global, e pior estado geral de saúde.

\section{REFERÊNCIAS BIBLIOGRÁFICAS}

Atanázio, E. A., Santos, J. M., Dionísio, L. R., Silva, J. \& Saldanha, A. W. (2013). Vulnerability to alcohol use: a study with adolescents from the public and private school systems. SMAD - Revista Eletrónica de Saúde Mental, Álcool e Drogas, 9 (1), 11-17. Disponível em: http://bases.bireme.br/cgi-bin/wxislind.exe/iah/ online $/$ ? IsisScript $=\mathrm{iah} / \mathrm{iah} . \mathrm{xis} \& \mathrm{src}=$ google\&base $=$ LIL ACS\&lang $=$ \&\&nextAction $=\operatorname{lnk} \&$ exprSearch $=702002$ \&indexSearch=ID

Bor, J. S. (2015). Among the elderly, many mental illnesses go undiagnosed. Health Affairs, 34 (5), 727-731. Disponível em: https://doi.org/10.1377/ hlthaff.2015.0314

Chong, S. A., Abdin, E., Picco, L., Pang, S., Jeyagurunathan, A., Vaingankar, J. A., Kwok, K. W. \& Subramaniam, M. (2016). Recognition of mental disorders among a multiracial population in Southeast Asia. BMC Psychiatry, 16, 121. Doi: 10.1186/s12888-0160837-2

Faria, C. S. \& Martins, C. B. G. (2016). Violência entre adolescentes escolares: condições de vulnerabilidade. Enfermería Global, 42, 171-184. Disponível em: http://scielo.isciii.es/pdf/eg/v15n42/pt_docencia2.pdf
Gulliver, A., Griffiths, K. M., \& Christensen, H. (2010). Perceived barriers and facilitators to mental health help-seeking in young people: a systematic review. BMC Psychiatry, 10 (113), 1-9. Disponível em: https:// doi.org/10.1186/1471-244X-10-113

Jorm A. F. (2000). Mental Health Literacy: Public Knowledge and beliefs about mental disorders. British Journal of Psychiatry, 177, 396-401. doi: 10.1192/ bjp.177.5.396

Jorm, A. F. (2012). Mental health literacy: Empowering the community to take action for better mental health. American Psychologist, 67, 231-243. doi: $10.1037 / \mathrm{a} 0025957$

Jorm, A. F., Wright, A., \& Morgan, A. (2007). Where to seek help for a mental disorder? National survey of the beliefs of Australian youth and their parents. The Medical Journal of Australia, 187, 556-560. Disponível em: https://www.ncbi.nlm.nih.gov/pubmed/18021042

Loureiro, L. (2013a). Estigma pessoal e percebido acerca do abuso de álcool e intenção de procura de ajuda. Revista de Enfermagem Referência, 3 (11), 59-66.

Loureiro, L. (2013b). Barreiras e determinantes da intenção de procura de ajuda informal na depressão. International Journal of Developmental and Educational Psychology, 1 (1), 733-746.

Loureiro, L., Jorm, A. F., Mendes, A., Santos, J., Ferreira, R., \& Pedreiro, A. (2013a). Mental health literacy about depression: a survey of Portuguese youth. BMC Psychiatry, 13 (1), 129. Disponível em: https://doi. org/10.1186/1471-244X-13-129

Loureiro, L. M. J., Barroso, T. M. M. D. A., Mendes, A. M. O. C., Rodrigues, M. A., Oliveira, R. A. A. N., \& Oliveira, N. R. N. (2013b). Literacia em saúde mental de adolescentes e jovens portugueses sobre abuso de álcool. Escola Anna Nery Revista de Enfermagem, 17 (3), 474-481.

Picco, L., Seow, E., Chua, B. Y., Mahendran, R., Verma, S., Chong, S.A. \& Subramaniam, M. (2017). Recognition of mental disorders: findings from a cross-sectional study among medical students in Singapore, BMJ Open, 7 (12). Doi: 10.1136/bmjopen-2017-019038 
Reavley, N. J., \& Jorm, A. F. (2011). Young people's recognition of mental disorders and beliefs about treatment and outcome: Findings from an Australian national survey. The Australian \& New Zealand Journal of Psychiatry, 45 (10), 890-898. Doi: 10.3109/00048674.2011.614215

Ribeiro, K. C. S., Pereira, L. B., Wiesl, I. R. B., Silva, J., \& Saldanha, A. A. W. (2017). Consumo de álcool e tabaco e associação com outras vulnerabilidades em jovens. Psicologia, Saúde \& Doenças, 18 (2), 348-359. Disponível em: http://dx.doi.org/10.15309/17psd180206

Rickwood, D., Deane, F., Wilson, C., \& Ciarrochi, J. (2005). Young people's help-seeking for mental health problems. Australian e-Journal for the Advancement of Mental Health, 4 (3) Suppl, 1-34. Disponível em: https://doi.org/10.5172/jamh.4.3.218

Rosa, A., Loureiro, L., \& Sequeira, C. (2016). Literacia em saúde mental sobre abuso de álcool em adolescentes: Desenvolvimento de um instrumento de medida. Revista Portuguesa de Enfermagem de Saúde Mental, (16), 59-68. Disponível em: http://dx.doi. org/10.19131/rpesm.0158
Tavares, M., Reinaldo, A., Villa, E., Henriques, B., \& Pereira, D. (2017). Perfil de adolescentes e vulnerabilidade para o uso de álcool e outras drogas. Revista de Enfermagem UEFP On Line, 11 (10), 3906-3912. Doi: 10.5205/reuol.12834-30982-1-SM.1110201727

Tomé, G., Matos, M. G., Gomes, P., Camacho, I., \& Gaspar, T. (2017). Promoção da Saúde Mental nas Escolas - Projeto ES'COOL. Revista de Psicologia da Criança e do Adolescente, 8 (1), 173-184. Disponível em: http://revistas.lis.ulusiada.pt/index.php/rpca/article/view/2485

Wandekoken, K. D., Vicente, C. R., \& Siqueira, M. M. (2011). Alcoolismo parental e fatores de risco associados. Revista Eletrónica de Saúde Mental. Álcool e Drogas, 7 (3), 161-167. Disponível em: https://www. revistas.usp.br/smad/article/view/49590/53665

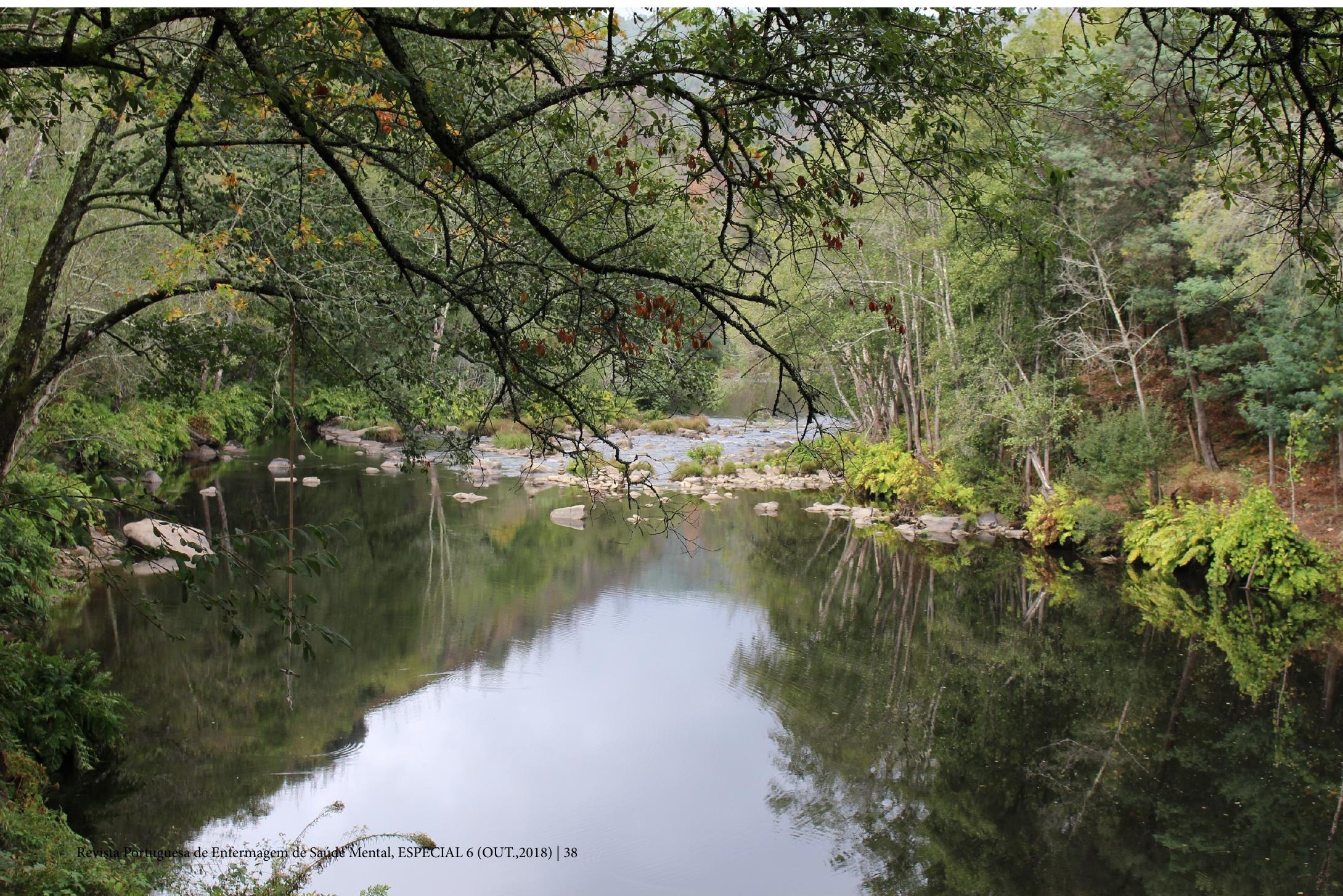

